\title{
THE CORRELATION BETWEEN KINEMATICAL PROPERTIES AND AGES OF STELLAR POPULATIONS
}

\author{
J. EINASTO
}

W. Struve Astrophysical Observatory, Tôravere, Estonia, U.S.S.R.

The spatial and kinematical properties of galactic populations are very conservative for time changes. Therefore the study of these properties gives us certain information on the past dynamical evolution of the Galaxy, in particular on the evolution of star generating medium (interstellar gas, as generally accepted). The detailed study of spatial structure of stellar populations in our Galaxy is possible in exceptional cases only. But the study of kinematical properties is possible practically for all populations, which makes these studies very useful for cosmogonic purpose.

In order to obtain adequate quantitative information for the study of dynamical history of the Galaxy the statistical data on stellar velocities are to satisfy the following requirements: populations under study must be physically homogeneous; statistical samples of stars must be free from selection effects, especially from velocity selection; information on r.m.s. errors of observed quantities must be known in order to correct the results for accidental observational errors; the data for the determination of the age of the sample must be available.

We have collected published data on stellar velocities and determinations of kinematical parameters back to the fundamental work by Parenago (1951). A critical analysis of these data shows, however, that only a part of the available data can be used for our purpose. For all populations mean velocity dispersion $\sigma=\frac{1}{3}\left(\sigma_{\mathrm{R}}^{2}+\right.$ $\left.+\sigma_{\theta}^{2}+\sigma_{z}^{2}\right)^{1 / 2}$ and mean heliocentric centroid velocity in rotational direction, $\bar{V}_{\theta}$, have been calculated $\left(\sigma_{R}, \sigma_{\theta}, \sigma_{z}\right.$ are the velocity dispersions in galactic cylindrical coordinates). The velocity dispersions have been corrected for observational errors using a method, proposed by us (Einasto, 1955). The age of populations has been determined from Iben's evolutionary tracks. For halo populations the individual age determinations coincide within possible errors. The relative age of these populations has been estimated theoretically, adopting for oldest halo population the age of the Galaxy, $10^{10} \mathrm{yr}$, and for other halo populations an age, needed for the population considered to collapse with free fall acceleration to its observed dimensions.

The Strömberg diagram for populations studied is given in Figure 1. Populations with metal deficit are represented by open circles, populations with normal metal content by points, the interstellar gas by a cross. The smooth curve shows the mean dependence between $\sigma$ and $\bar{V}_{\theta}$ of populations of different ages; the latter is indicated in $10^{9} \mathrm{yr}$, starting from the formation of oldest galactic populations known.

The main results of this study may be formulated as follows.

(1) If we attribute all metal deficient subpopulations to the halo, then it appears that the halo is rather heterogeneous in its kinematical properties: it contains all subpopulations with velocity dispersion $\sigma \geqslant 50 \mathrm{~km} \mathrm{~s}^{-1}$. The corresponding axial ratio 


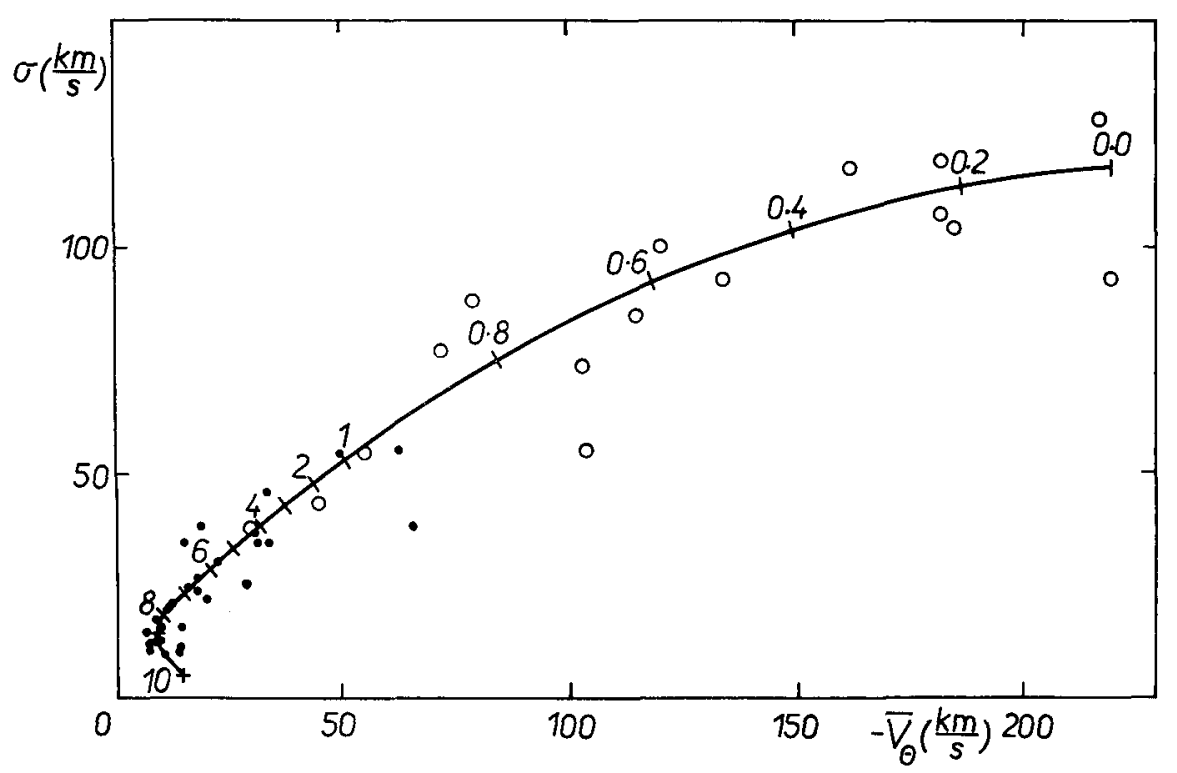

Fig. 1. The Strömberg diagram for populations. The numbers give the birthdates in $10^{9} \mathrm{yr}$ starting from the formation of the oldest populations.

$\varepsilon$ of equidensity ellipsoids, calculated from our recent model of the Galaxy (Einasto, 1970), is equal to or larger than 0.10. Studying the structure of the Andromeda galaxy M31 we also came to the conclusion that its halo consists of a mixture of subpopulations with $\varepsilon \geqslant 0.10$ (Einasto, 1972).

These results show that intermediate subsystems of the Galaxy according to Kukarkin (1949) also belong to the halo.

(2) Direct age determinations of stellar populations are too inaccurate to estimate the duration of the initial galactic collapse. There exists, however, indirect observational (Sandage, 1969) and theoretical (Eggen et al., 1962) evidence that the collapse proceeded in a short time scale compared with the age of the Galaxy.

(3) The populations of the galactic disc have $15 \leqslant \sigma \leqslant 50 \mathrm{~km} \mathrm{~s}^{-1}$ and, respectively $0.02 \leqslant \varepsilon \leqslant 0.10$ (Einasto, 1970). The age dependence of spatial and kinematical properties of these populations may be caused by the action of irregular gravitational forces (Spitzer and Schwarzschild, 1953; Kuzmin, 1961).

(4) The subsystems of interstellar gas and young stars rotate with a velocity, smaller than the circular one. Therefore the young stellar subsystems are nonsteady and time is needed for them to obtain steady structure. This result supports the recent discovery of the non-stationary state of young populations by Dixon (1967a, b, 1968) and Jôeveer (1968).

\section{References}

Dixon, M. E.: 1967a, Monthly Notices Roy. Astron. Soc. 137, 337.

Dixon, M. E.: 1967b, Astron. J. 72, 429.

Dixon, M. E.: 1968, Monthly Notices Roy. Astron. Soc. 140, 287. 
Eggen, O. J., Lynden-Bell, D., and Sandage, A. R.: 1962, Astrophys. J. 136, 748.

Einasto, J.: 1955, Tartu Astron. Obs. Publ. 33, 35.

Einasto, J.: 1970, Tartu Astron. Obs. Teated 26, 1.

Einasto, J.: 1972, Tartu Astron. Obs. Teated 40, 3.

Jôeveer, M.: 1968, Tartu Astron. Obs. Publ. 36, 84.

Kukarkin, B. V.: 1949, The Investigation of the Structure and Evolution of Stellar Systems on the Basis of the Study of Variable Stars, Gosud. Izd. Tekhniko-Theoreticheskoj Lit., Moscow.

Kuzmin, G. G.: 1961, Tartu Astron. Obs. Publ. 33, 351.

Parenago, P. P.: 1951, Trudy Astron. Inst. Sternberg $20,26$.

Sandage, A.: 1969, Astrophys. J. 157, 515.

Spitzer, L. and Schwarzschild, M.: 19.53, Astrophys. J. 118, 106. 